\title{
Discussion of Online Teaching of Information Retrieval Course for International Economics and Trade Major
}

\author{
YIN Hong-lei ${ }^{1, a}$ JIANG Shu-zhu ${ }^{2, b^{*}}$
}

\author{
${ }^{1}$ Library, Shandong Technology and Business University, Yantai China \\ ${ }^{2}$ School of Economics, Shandong Technology and Business University, Yantai China \\ ayhljsz@163.com \\ b*99822710@qq.com
}

\begin{abstract}
Affected by COVID-19, universities can only teach online. In the online teaching process of information retrieval courses for international economics and trade majors, rationally plan the teaching content, select group training and micro-learning on teaching methods, and select cloud classes and Dingding for teaching software. The solutions to the problems of network congestion, increased workload of teachers, difficult supervision and difficulty in time control in online teaching are given.
\end{abstract}

Keywords: information retrieval, online teaching, micro-lessons, group training

\section{国际经济与贸易专业信息检索课程在线教学探讨}

殷红蕾 $1, \mathrm{a}$ 姜书竹 $2, \mathrm{~b}^{*}$

\author{
${ }^{1}$ 山东工商学院图书馆, 烟台, 中国 \\ ${ }^{2}$ 山东工商学院经济学院, 烟台, 中国 \\ ayhljsz@163.com \\ b*99822710@qq.com
}

\section{摘要}

2020年上半年，受新冠疫情影响，高校只能进行在线教学。在国际经济与贸易专业信息检索课程的在线教学过 程中, 需合理规划教学内容, 教学方法上选择分组训练和微课, 教学软件选择云班课和钉钉。并针对在线教学 过程中遇到的网络拥堵、教师工作量增加、监管不易和课堂教学时间难以控制等问题都给出了解决的办法。

关键词: 信息检索、在线教学、微课、分组训练

\section{1. 引言}

受新冠肺炎疫情影响，2020年春季学期开始，国 内高校几乎都不允许学生返校, 只能开展在线教学, 后来疫情有所缓解之后, 虽然很多高校陆续允许学生 返校, 但在线教学仍然占据重要地位。移动互联网和 教学软件平台的发展为开展在线教学活动提供了坚 实的物质基础。教育部要求中国大学慕课网等在线教 学平台在疫情期间, 向高校免费开放 2 万多门的课程 资源。教师可以使用已经建成并投入使用的在线课程 资源, 也可以采用直播的方式或自建课程, 结合使用
在线教学软件平台和社交软件, 可以有效解决自建课 程的在线教学问题。毕业论文和学年论文是非常重要 的教学环节, 尤其毕业论文是每次教学评估重点检查 的内容, 因此一定要保证毕业论文的质量。而文献检 索和数据收集是国际经济与贸易专业学生写好论文 的基础。这就需要开设信息检索或专业论文写作课 程，系统地学习文献资料检索、数据收集。信息检索 是一门注重上机实践、操作性很强的课程, 特别适合 利用网络进行在线教学。本文仅以本校国际经济与贸 易专业为例, 探讨在疫情期间如何开展国际经济与贸 易专业的信息检索课程的在线教学。 


\section{2. 教学实施}

近些年，越来越多的高校实施了学分制改革，改 变以往按年度固定收费的做法, 改为按照学分收费。 与此同时, 毕业要求的总学分大幅减少, 同时作为山 东省高水平应用型专业, 按照省里要求, 实践学分比 重必须提高到 $25 \%$ 。国际经济与贸易专业信息检索课 程可在这样的背景下作为一门实验课程开设的, 以培 养学生的实践动手能力为主。对于该课程的在线教 学, 需要做好课程教学设计, 这主要是指要规划好教 学内容、教学方法、教学工具三个方面。

\section{1 教学内容}

这门课程的教学内容方面, 总课时一般可设置为 16 课时, 所以不能够做到面面俱到, 不能够“麻雀虽 小, 五脏俱全”。教学内容必须有侧重点, 着重讲解 那些核心的知识点, 非核心的、难度不大的知识点可 以留给学生自己在课前或课后自学, 等到他们在使用 过程中遇到问题时再进行答疑辅导。该课程教学是针 对国际经济与贸易专业学生的, 因此有别于一般的信 息检索课程。如表1所示, 国贸专业信息检索课程的 主要内容既包括信息检索的概念、原理与检索技巧, 中英文文献的检索与原文获取, 又包括经济贸易统计 数据的获取。

表1 教学内容与时间安排

\begin{tabular}{|c|c|c|c|}
\hline 周次 & 章 & 内容 & 课时 \\
\hline 1 & 信息检索初步 & 信息检索的概念、原理与检索技巧 & 2 \\
\hline 2 & 中文文献检索 & 知网、维普、万方、超星 & 4 \\
\hline 3 & 英文文献检索 & web of science、 EBSCO、Emerald、Elesevier & 4 \\
\hline 4 & ScienceDirect & 2 \\
\hline 5 & 原文获取 & 读秀、CALIS文献传递 & 2 \\
\hline 6 & 宏观经济数据 & trademap、comtrade & 2 \\
\hline
\end{tabular}

据库查询并获取英文文献; 介绍如何通过读秀和calis

其中信息检索初步主要是介绍信息检索的概念、 原理与检索技巧，计划用 2 个课时。

文献检索包括三部分内容: 中文文献检索主要介 绍和训练学生如何使用中国知网、维普、万方和超星 等在线平台获取科研期刊、学位论文和图书的电子文 档; 英文文献检索主要介绍如何利用web of science、

EBSCO、Emerland、Elesevier ScienceDirect等外文数 文献传递功能, 来获取那些只能查到标题和摘要等信 息、但无法直接获取原文的中外文文献。

国际贸易统计数据主要介绍中国农业部网站和 联合国下属的两个数据库 trademap和comtrade; 宏观 统计数据主要介绍世界银行的WDI数据和中国国内 的统计年鉴（国家统计局和地方统计局网站），详情 如表2所示。

\section{表2 常用贸易统计数据来源网站}

\begin{tabular}{|c|c|c|l|}
\hline 序号 & 数据类型 & 主办方 & \multicolumn{1}{|c|}{ 网址 } \\
\hline 1 & $\begin{array}{c}\text { 世界各国经济社会发展数据 } \\
\text { (WDI) }\end{array}$ & 世界银行 & https://databank.worldbank.org/home.aspx \\
\hline 2 & $\begin{array}{c}\text { 世界各国国际贸易进出口统计 } \\
\text { (Comtrade) }\end{array}$ & 联合国 & https://comtrade.un.org/data/ \\
\hline 3 & $\begin{array}{c}\text { 世界各国国际贸易进出口统计 } \\
(\text { Trademap) }\end{array}$ & $\begin{array}{c}\text { 联合国贸发会议国际贸易 } \\
\text { 中心 }\end{array}$ & https://www.trademap.org/ \\
\hline 4 & 中国历年经济数据 & 中国国家统计局 & https://data.stats.gov.cn/ \\
\hline 5 & $\begin{array}{c}\text { 中国农业生产和农产品贸易相 } \\
\text { 关数据 }\end{array}$ & 中国农业农村部 & http://zdscxx.moa.gov.cn:8080/nyb/pc/index.jsp \\
\hline
\end{tabular}

\section{2 教学方法}

本课程不采用传统以教师讲授为主的教学方法, 而是根据课程本身的特点和网络教学优势, 计划采用 分组训练和微课两种方法实施翻转课堂教学。

\subsection{1分组训练}

在本课程教学中, 第一堂课就将学生分成每组5-6 人的学习小组, 很多教学活动分组进行。首先, 由教 师在课堂上组织学生就某个问题进行分组讨论或分 组训练; 其次, 课后任务也分组完成, 要求每组学生 对任务进行充分讨论并分工合作完成之后再提交。例 如，在练习贸易数据查询时，教师先进行数据库的简 单讲解, 然后布置小组任务, 第一步让学生查询某个 国家的对外贸易情况, 包括进出口总额和与各贸易伙 伴的双边贸易额情况，第二步，要求查询某国某种产 品的进出口情况，包括贸易额、贸易量和单价等数据。 
学生就会在小组中先商量这个数据要到哪个网站查 询, 后续怎么分工操作? 在实际操作中又会发现需要 了解产品的HS编码是什么? 产品的HS编码这个问题 可以通过百度解决, 然后再回到相应统计网站一步一 步地进行查询。在新冠疫情期间, 只能在线教学的情 况下, 分组教学将面临一些问题。学生之间因只能在 网上通过社交软件或教学平台进行讨论, 讨论的积极 性可能不高, 有些学生可能希望搭便车, 这就需要严 格要求小组任务分解到每个小组成员, 使每个小组成 员都有事可做, 同时需要教师积极推动并进行严格的 监督和考核。如果教学软件能实现在线的分组讨论, 效果应该更好一些。

\subsection{2微课教学}

已有研究表明, 课堂教学开始时学生能够持续认 真听讲15到20分钟, 再往后学生注意力集中的时间会 越来越短, 到课堂结束时只能维持三五分钟。因此, 如果是采用满堂灌的教学方式, 上课的效果肯定不太 理想。所以需要把这个课程的内容按照知识点进行分 解, 然后重新安排教学内容。可以把每个数据库或搜 索引擎的功能和使用拆解成单个知识点, 由教师做一 下简单介绍, 剩余时间交给学生练习, 让学生在实际 操作中去发现和了解细节问题, 教师再适时给予指 导。建议每个知识点讲授的时间尽量控制在 10 分钟以 内, 然后安排答疑、练习、讨论或分享等教学活动, 这样学生就不会感觉太疲㤂。相应的, 为了方便学生 自学和课后复习, 可以录制教学视频, 应尽量录制短 视频, 然后辅以适当的练习和思考。在具体到数据库 功能和使用介绍时, 比如中国知网, 可以先打开CNKI 主页, 简单介绍CNKI的资源, 然后让学生汶览略微 熟悉一下CNKI, 再按不同的检索方法进行讲解和训 练, 保证学生边学边练, 在学生的实际检索过程中教 会学生浏览检索结果技巧, 从而学会如何查找和筛选 资源，以及如何发现该领域的研究热点和前沿问题。

\section{3教学软件}

在线教学必须借助网络及相关的软件和硬件设 备, 所以这里的教学工具应包括软件和硬件两大方 面。如果学校提供足够的经费支持和帮助, 软件和硬 件都可以选最好的, 不过目前绝大多数学校最多也就 是帮助协调在线教学平台, 获取课程资源, 其他的软 硬件和网络都需要教师自己解决。硬件设备主要是能 上网的电脑, 教师家里基本都有, 所以需要解决的主 要是软件问题, 软件的选择至少包括三个方面: 教学 软件平台、即时通讯工具和视频录制软件。

\section{3. 1 教学软件平台}

为了让学生可以在课前课后系统地学习和复习, 最好是选择一个在线教学平台, 进行课程建设。教师 把整门课程的内容按照章节成体系的上传到选定的
教学平台上, 以便学生随时查看。本课程选用的云班 课这个平台, 其优势有三: 第一, 无须使用VPN登录 校园网, 可以使用智能终端直接联网登录, 几乎所有 大学生都有智能手机, 下载APP即可使用。第二, 云 班课功能相对简洁, 方便教师熟悉软件使用, 但是能 满足大部分的教学需要。第三, 提供智能化教学过程, 学生所有的学习活动都会留下痕迹, 并获得经验值, 教师可以据此判断学生参与学习的情况。既可以经验 值总数作为平时成绩的依据, 也可以导出各类活动的 经验值, 按照事先规定的项目给平时成绩。教师先在 云班课平台上注册账号, 开建课程, 把课程号发给学 生, 让学生自己加入。然后, 再逐步把课程内容准备 好, 上传到平台上; 如果内容已经很成熟, 也可以一 次性把课程内容都准备好。课程建设需要在电脑上登 录网页完成, 上课时可以在手机端和电脑端同时进 行。

\section{3. 2 即时通讯工具}

开展在线直播教学, 只有教学软件是不够的, 还 需要配合即时通讯工具, 组建课程群, 如 $\mathrm{QQ}$ 群、微 信群或钉钉群。这些课程群非常重要, 是师生交流的 重要工具, 可以用于课前的通知、课上的讨论、课后 的答疑、甚至课程直播。钉钉和 $\mathrm{QQ}$ 提供了专业的课 程群功能, 教师在建群时如果选择了课程群的模式, 就可使用签到、作业、分享文件等功能, 也可以直播 或以视频会议的方式授课、与学生进行互动。此外, 还有其他一些直播软件或会议软件可以选用, 不过需 要单独下载手机APP或电脑客户端。在疫情期间, 所 有学校都进行在线教学, 网络拥堵现象时有发生, 此 时选择教学平台和即时通讯工具的重要标准之一是 不卡顿。

\subsection{3视频录制软件}

为了方便学生课前自学, 需要提前录制教学视 频。录制短视频是年轻人比较喜欢的事情, 也有很多 优秀软件如抖音等。但是教学视频录制很多时候需要 配合PPT, 所以屏幕录制软件应该更合适一些。如果 用手机录制可以考虑使用录屏精灵软件, 开启录屏精 灵软件后, 选择横屏或坚屏, 然后在手机上打开PPT, 点击“录制”就可以开始录制了。一般的课件录屏, 完 全可以在手机上进行, 但是有些内容只能在电脑上演 示。这就需要电脑录制, 需要有麦克风才行, 笔记本 都自带麦克, 只有台式机需要单独配备麦克风, 软件 可以选视频编辑王, 没有录制时长的限制, 还可以进 行编辑。当然, 为了取得更好的教学效果, 若学校能 采购高质量的耳麦和正版专业视频录制和编辑软件 更好。

\section{3. 线上教学可能遇到的问题与解决办法}

疫情期间的全面网络教学是不得已而为之, 不仅 教师们还没有做好准备, 而且各类服务商也没有做好 
准备。开展在线教学活动的同时, 还进行教学方法上 的改革, 所以难免存在诸多问题, 需要重视并加以解 决。

\section{1 网络拥堵问题}

疫情期间开展线上教学最突出的问题就是网络 拥堵严重。这是有史以来第一次, 从大学到中小学, 所有的学生同时进行网络学习。一时间各大在线教学 平台的服务器访问人数激增, 高校刚开学那几天, 包 括中国大学慕课、雨课堂、云班课等平台都曾经发生 严重拥堵，甚至无法登录。这个问题现在已经有明显 改善, 但网络信号不佳的情况仍时有发生, 同样影响 网课学习质量。QQ群和钉钉群等在直播时也遇到此 类问题, 经常有学生反映卡顿严重, 只好退出再进入 会议或直播, 但是问题依旧存在。

\section{2教师工作量明显增加}

在线教学势必会大幅度地增加教师的工作量, 耗 费教师更多的时间和精力。尤其是疫情期间, 在完全 没有准备、也没有资金投入的情况下，需要在很短时 间内建设一门在线课程, 压力很大。且多数教师承担 两门以上课程, 这种情况下, 教师需要独立完成国贸 专业的信息检索这门在线课程建设, 实属不易。除了 录制视频、上传PPT和其他学习资料以外, 还需要答 疑和批改作业。虽然可以从网上获取一些资源, 但是 这些资源质量参差不齐, 需要花费时间进行整理、修 订。云班课等软件允许学生在线提交作业, 如果学生 数量多, 批改作业的工作量就非常大, 一个周可能有 几百份作业需要批改。教师需要独立完成所有工作, 非常不易。

\section{3课堂监管不易}

在线教学过程中, 借助软件, 很容易查看出勤情 况, 但无法判断电脑前面或看着手机屏幕的学生正在 做什么。他们到底是在认真听课, 还是在刷电视剧、 玩游戏、做其他事情? 在线讲授过程中最好频繁的进 行互动, 但经常会发现有些学生“失联”, 课后告诉教 师, 网络不好, 听不见, 所以没反应。至于有没有认 真听讲, 更是无法直接观察了。因此, 加强课堂监管 刻不容缓。另外, 分组讨论时, 有点小组大部分同学 积极性不高, 积极发言的同学较少。

\section{4课堂时间难以控制}

课堂互动的时间不太好掌控, 尤其是当前在线教 学过程中, 可能因为网络延迟, 点名之后过一会, 学 生才回应, 浪费一些时间; 也可能讨论过于热烈, 停 不下来。结果是互动的时间超出预期, 导致其它的一 些教学环节不能按计划完成。而每次课都有相应的计 划, 所以也不能每次都往后推, 占用后面的时间。

\section{4. 线上教学所面临问题的解决办法}

\section{1 充分利用云班课平台功能解决网络拥堵问 题}

应该充分利用云班课平台功能，破解网络拥堵问 题。教师可将自己录制的教学视频上传到云班课的资 源里, 方便学生课前或课后观看; 对于在线的慕课资 源, 也要求学生尽量提前或课后看。另外, 对于课堂 讨论和作业, 可以借助云班课的轻直播功能, 在轻直 播里进行讨论和讲解, 这样既不怕卡顿, 也能把过程 保存下来, 方便学生日后复习。当然, 作业讲解, 也 可以在 $\mathrm{QQ}$ 群、钉钉群里进行, 也能留下痕迹, 只是 时间久了, 累积的内容太多, 不方便查看。

\section{2多措并举解决教师工作量增加问题}

对于在线教学导致教师工作量明显增加的问题, 可从以下三个方面解决: 第一, 教师要想方设法自己 慢慢建设在线课程, 建成之后可以继续使用, 后续维 护会相对简单, 日后的教学活动就会轻松很多; 第二, 答疑可以在 $\mathrm{QQ}$ 群里或云班课的轻直播里进行, 所有 学生都可以看到问答过程, 这样同一个问题就不会有 学生重复提问, 从而节省了重复答疑的时间; 第三, 对于作业可以考虑分组完成, 减少批改的份数, 但要 保证所有人参与, 或者可以在学生都提交之后, 把答 案发给学生来互评。为了避免分组作业中可能出现的 搭便车行为, 要求先个人做完, 再由组长召集讨论, 或安排轮值组长, 同时老师要关注讨论情况。此外, 为提高教师开展在线教学积极性, 学校层面应该出台 相关政策, 对于在线课程建设和使用在线课程资源授 课的教师予以必要的资金支持或对相关工作计算工 作量。

\section{3以多种形式的教学互动实现课堂监管}

针对在线课程的课堂监管困难, 可以采取以下措 施：每次课限时签到，但签到不固定时间，可能在第 一或第二节开始，也可能是在下课之前; 每次课都准 备一些小问题随机找学生回答, 或是单个题目的小测 验, 要求限时完成; 分组讨论时, 要求每个学生都必 须发言, 经过组内充分讨论后, 推荐一名学生代表本 组发表意见, 组内讨论通过设置分组的轻直播完成, 教师可以随时查看讨论情况; 也可就某个问题开展头 脑风暴, 让所有学生都开动脑筋, 把自己的想法或答 案通过云班课的头脑风暴功能发表，等教师结束该活 动时, 所有学生都可查看。总之, 要想使学生尽量参 与到课堂中来, 就需要多增加互动环节。另外, 对于 小组讨论不积极的问题, 教师要适当参与到小组讨论 中来, 多关注、鼓励; 另外可以要求课后讨论在云班 课或QQ群中进行, 以便于教师查看讨论情况。 


\section{4教师需要提高掌控课堂的能力}

针对在线教学过程中时间不够用的问题，一方面 教师需要提高掌控课堂的能力, 合理设计每堂课的内 容, 既要保证课堂讨论热烈进行, 又要把握时间, 力 保计划的内容都能按时完成。另一方面, 要充分利用 课外时间, 如果还有个别学生有疑问, 为了节省课堂 时间, 课后学生可以通过钉钉、云班课提问, 教师进 行单独辅导。

\section{5. 结论}

综上所述，在移动互联网已经普及的大背景下， 一些需要进行线上训练的课程比较适合进行在线教 学。信息检索就是一门比较适合开展在线教学的课 程, 虽然其在线教学过程中存在一些问题, 但只要能 解决其面临的问题, 就能保证在线教学的顺利开展。 并且, 在疫情结束、学生返校之后, 依然可以使用已 经建立起来的课程平台资源, 依托移动互联网开展线 上线下混合教学, 为学生撰写学年论文和毕业论文打 下良好的基础。

\section{项目基金}

本文为山东省高等学校本科教学改革项目《移动 互联网背景下应用型专业(群)教学生态系统建构研究: 知识分享与价值共创二重理念视角》（M2018X180） 的阶段性成果之一。

\section{REFERENCES}

[1] Zhang, D. (2016) A Study of the Teaching Mode for Literature Retrieval Courses in Colleges and Universities. Future and Development., 40:85-89.

[2] Han, S., Wang H. (2020) Research on the key links and effective methods of the implementation of online and offline hybrid teaching model. Wireless Internet Technology., 17: 99-101+106.

[3] Yan, B., Wang, D. (2019) Digital Literacy Education and Enlightenment of Public Library in Ameirca Under the Perspective of Poverty Alleviation Via Information. New Century Library., 21:85-90.

[4] Gao, S., Fan, S. (2019) Practice and Revelation of Digital Literacy in the Library of Queensland University, Australia. Research of Library Science., 22:95-101. 